THE KURUME MEDICAL JOURNAL

Vol. 41, p. 117-121, 1994

\title{
Genotype-Phenotype Agreement of Aldehyde Dehydrogenase 2 in 120 Healthy Japanese
}

\author{
ATSUSHI NISHIYORI ${ }^{* \dagger}$, KATSUHIRO FUKUDA* ${ }^{*}$ KYOGO ITOH** \\ AND HIROHISA KATO ${ }^{\dagger}$ \\ Departments of Public Health ${ }^{*}$, Immunology ${ }^{* *}$ and Pediatrics and Child \\ Health $^{\dagger}$, Kurume University School of Medicine, Kurume, 830 Japan
}

Received for publication June 28, 1994

\begin{abstract}
Summary: An individual genotype of aldehyde dehydrogenase 2 was observed using nail clippings by an improved method consisting of DNA extraction with guanidium thiocyanate, genomic DNA amplification by nonisotopic polymerase chain reaction, and gel electrophoresis of the DNA fragment. The results were consistent with those obtained using the corresponding peripheral blood samples. The individual phenotype was also determined by the Tokyo-University ALDH2-Phenotype Screening Test. Both types of ALDH2 were observed in 120 healthy Japanese and $48 \%$ were the typical or normal homozygote, $43 \%$ were the heterozygote, and $9 \%$ were the atypical or mutant homozygote of ALDH2. Among 57 individuals of both sexes with the typical homozygote, $88 \%$ were the phenotype of a non-flusher. Similarly $76 \%$ of the 63 heterozygote/atypical homozygotes had the phenotype of a flusher.
\end{abstract}

Key words: genotype - phenotype - aldehyde dehydrogenase 2 Japanese - polymerase chain reaction

\section{Introduction}

Ingested alcohol is metabolized to acetaldehyde by alcohol dehydrogenase $(\mathrm{ADH})$, which is then metabolized to acetic acid by aldehyde dehydrogenase (ALDH). ALDH2 which has a low $\mathrm{Km}$ value is regarded as the critical ALDH isoenzyme that determines an individual's intolerance to alcohol. Genotyping or phenotyping of ALDH2 is essential for genetic, clinical, or epidemiological studies of alcohol-related diseases. Since it is not always feasible to collect blood samples; other biological materials, such as scraped mucous membranes, urine, hair roots or fingernails can be used. Gene analysis using fingernail DNA has been reported (Kaneshige et al. 1992) but the proteinase $\mathrm{K}$ method is rather time consuming and may fail to extract DNA from manicured or smudgy nails as exemplified by Fig.1A. Phenotyping of an individual's ALDH2 can be performed by the Tokyo-University ALDH2-Phenotype Screening Test (TAST) which is a questionnaire consisting of 13 items (Yamada et al. 1988), and it may be used as an alternative measure for the genotype. Therefore, agreement between the two types is to be elucidated in human populations by race or other relevant factors. 

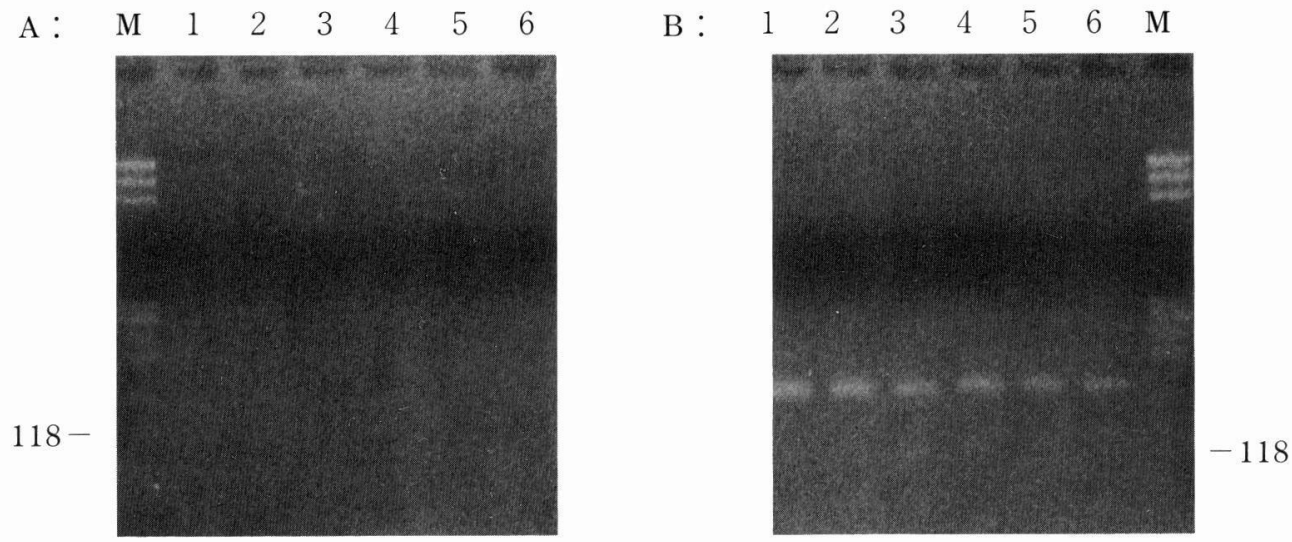

Fig. 1. A gel electropherogram of one-tenth of the PCR products of a $135 \mathrm{bp}$ DNA fragment containing the ALDH2 exon 12 from fingernails. The size marker is $\psi \mathrm{X} 174 / \mathrm{Hae}$ III (M). A: The DNA fragment was not detected in 3 manicured (lanes 1-3) and 3 smudgy nails (lanes 4-6) by the proteinase K method. Applying half of the same PCR products to the same electrophoresis also failed to yield a DNA fragment by the proteinase K method (data not shown). B: The DNA fragment was detected in the corresponding 3 manicured (lanes 1-3) and 3 smudgy nails (lanes $4-6$ ) by the guanidium method.

The present paper describes an improved ALDH2 genotyping method using nail clippings that yields results consistent with peripheral blood samples, and that agrees with the corresponding phenotypes of healthy Japanese.

\section{Materials and Methods}

Nails were collected using an ordinal nail clipper which was cleaned with $70 \%$ ethanolic cotton pads. The nails were cut into pieces of approximately $1-2 \times 5$ $10 \mathrm{~mm}$, and $10-20 \mathrm{mg}$ of the nail clippings were rinsed once with $1.0 \mathrm{~N} \mathrm{NaOH}$ and twice with distilled water in a $1.5 \mathrm{ml}$ micro test-tube. After adding $300 \mu \mathrm{l}$ of 4 M guanidium thiocyanate (Chomczynski and Sacchi, 1992), 0.5\% sodium N-lau- roylsarcosine, $25 \mathrm{mM}$ sodium citrate and 0.1 M 2-mercaptoethanol, the sample was incubated at $\mathrm{pH} 7.0$ at room temperature for $30 \mathrm{~min}$. Nucleic acids were extracted with phenol/chloroform and precipitated with ethanol and sodium acetate. After centrifugation the pellet was washed in $70 \%$ ethanol and the dried pellet was dissolved in $20 \mu \mathrm{l}$ of distilled water. One-tenth of the genomic DNA was subjected to 30 cycles of amplification by polymerase chain reaction (PCR) (Takeshita et al. 1993). 5$15 \mu \mathrm{l}$ of the PCR products were digested by Ksp 632 I to solely cut the 135 bp DNA fragment of a typical homozygote containing ALDH2 exon 12 into fragments of $112 \mathrm{bp}$ and $23 \mathrm{bp} .5 \mu \mathrm{l}$ of the PCR products were analyzed in $4 \%$ agarose gel, visualized by ethidium bromide staining and photographed under 
UV light.

Although disposable nail clippers are preferable for sampling to prevent DNA contamination, the DNA contamination due to the use of a single nail clipper for collecting many nails was examined by processing homozygous nails after heterozygous nails using a single clipper being cleaned as stated above.

The recovery of DNA was determined as follows. $480 \mu$ l of Tris-EDTA buffer was added to the DNA solution, and this was mixed with RNase A solution (final concentration of $20 \mu \mathrm{g} / \mathrm{ml}$ ) and incubated at $37^{\circ} \mathrm{C}$ for $30 \mathrm{~min}$. The DNA was extracted as above and precipitated with isopropylalcohol and sodium acetate. The purified DNA concentration was measured by absorbance at $260 \mathrm{~nm}$.

Individual finger nail clippings were collected by the method described above from 7 healthy males and 5 healthy females on the medical staff, aged 25-55, and peripheral blood samples were also collected from 10 of them. Finger nail clippings were collected by the same method from 92 healthy male and 28 healthy female medical students in Kurume University School of Medicine, who were aged 21-40, and who also responded individually to the TAST.

\section{Results}

Extraction of genomic DNA and ALDH2 genotyping were successfully performed with 132 fingernail clippings including 10 manicured and smudgy nails. Figure 1B shows the DNA fragments extracted by the guanidium method from 3 manicured and 3 smudgy nails which had no DNA detectable by the proteinase $\mathrm{K}$ method. The results of ALDH2 genotyping of the 10 samples completely matched the typings based on the corresponding peripheral blood.

Figure 2 shows that the processing of 2 to 3 heterozygous nails did not contaminate the subsequent ALDH2 genotyping of 4 atypical or mutant homozygotes (lanes 1-4), which have the $135 \mathrm{bp}$ DNA fragment containing the ALDH2 exon 12 , and of 2 typical or normal homozygotes (lanes 5-6) which have the 112 bp DNA fragment. The $23 \mathrm{bp}$ DNA fragment is not visible on the electropherogram.

The mean recovery of DNA in the 10 nail samples was $0.33 \mu \mathrm{g} / 10 \mathrm{mg}$ nail.

The composition of the 3 genotypes, the 2 phenotypes and the genotype-phenotype agreement are shown in Table 1. The proportions of typical or normal homozygote, heterozygote, and atypical or mutant homozygote were $45 \%$ (41/92),

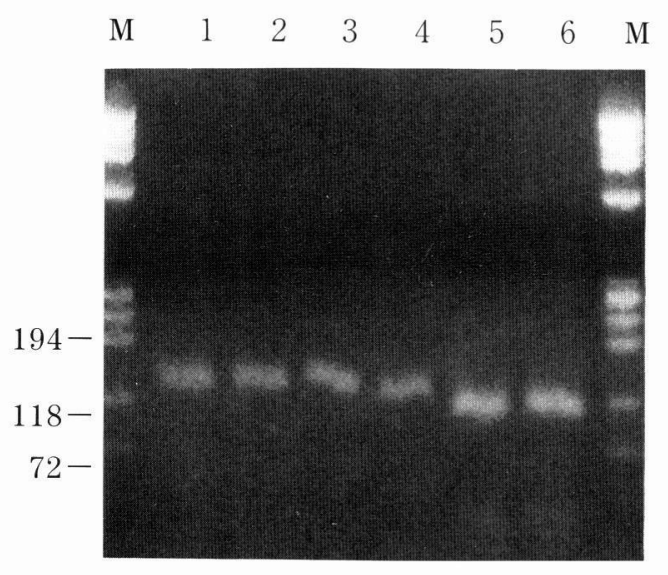

Fig. 2. A gel electropherogram showing no contamination by the previous processing of heterozygous nails for ALDH2 genotyping on 4 atypical or mutant homozygotes (lanes 1-4) and on 2 typical or normal homozygotes (lanes 5-6). 
TABLE 1.

Genotype-phenotype agreement of aldehyde dehydrogenase 2 in 120 healthy Japanese

\begin{tabular}{|c|c|c|c|c|c|c|c|}
\hline \multirow{3}{*}{ Genotype } & \multicolumn{7}{|c|}{ Phenotype observed by TAST* } \\
\hline & \multicolumn{2}{|c|}{ Male } & \multicolumn{2}{|c|}{ Female } & \multicolumn{3}{|c|}{ Both Sexes } \\
\hline & Non FL & $\mathrm{FL}^{* *}$ & Non FL & $\mathrm{FL}$ & Non FL & FL & Tota \\
\hline Normal homozygote & 35 & 6 & 15 & 1 & 50 & 7 & 57 \\
\hline Heterozygote & 12 & 34 & 1 & 5 & 13 & 39 & 52 \\
\hline Mutant homozygote & 0 & 5 & 2 & 4 & 2 & 9 & 11 \\
\hline Total & 47 & 45 & 18 & 10 & 65 & 55 & 120 \\
\hline
\end{tabular}

* TAST : Tokyo-University ALDH2-Phenotype Screening Test

${ }^{* *} \mathrm{FL}$ : alcohol flusher

$50 \%$ (46/92), and 5\% (5/92), respectively for the males. Those for female were $57 \%(16 / 28), 21 \%(6 / 28)$, and $21 \%(6 / 28)$, and those for both sexes were $48 \%$ (57/120), 43\% (52/120), and 9\% (11/120), respectively. The sensitivity of the TAST for differentiating a normal homozygote from the other 2 genotypes based on the non-flushing reaction would be $85 \%$ (35/41) and the corresponding specificity would be $76 \%(39 / 51)$ for the males. Similarly, the values for females were $94 \%(15 / 16)$ and $75 \%(9 / 12)$, respectively, and the values for both sexes were $88 \%$ (50/57) and 76\% (48/63), respectively.

\section{Discussion}

Polyacrylamide gel can be used instead of agarose for the electrophoresis, enabling the DNA fragment of $23 \mathrm{bp}$ to be visible on the electropherogram. However, it does not affect the ALDH2 genotyping.

The ALDH2 genotyping method described in this report is an improved method applicable not only to blood but also to nail clippings, and it yields consistent genotyping results. It may be more flexible for mass processing in human genetics or in epidemiological studies than the use of blood samples.

The proportion of the 3 genotypes for all the subjects in the present study is comparable to that previously reported (Takase and Takada, 1991), but there seems to be a discrepancy based on sex, which is probably due to the small size of the data sample.

The TAST might be influenced by an individual's drinking experience, particularly for females, and it could affect the results of the TAST. However, the sensitivity or specificity of the TAST observed in the present study is comparable to that previously reported based on more than 500 workers (Takeshita et al. 1994). A relatively small sample size and neglect of the $\mathrm{ADH}$ genotype might partially explain the differences.

Acknowledgments: The authors would like to thank Professor K. Morimoto and Associate Professor T. Takeshita, Osaka 
University School of Medicine and Professor M. Ogata, Sapporo Medical College for their suggestions during this study.

\section{References}

Сномczynski, P. and SAcchi, N. (1987). Single-step method of RNA isolation by acid guanidium thiocyanate-phenol-chloroform extraction. Anal. Biochem. 162, 156-159.

Kaneshige, T., Takagi, K., Nakamura, S., Hirasawa, T., SADA, M. et al. (1992). Genetic analysis using fingernail DNA. Nucleic Acids Res. 20, 5489-5490.

TAKASE, S. and TAKADA, A. (1991). Alcohol and acetaldehayde metabolism and liver injury. Taisha 28, 431-439. (in Japanese)
TAKeShita, T. and Morimoto, K. (1994). Genetic controlling factor of alcohol drinking behavior and its effect on physical health, the 7th report. J. Epidemiol. 4, 67-68. (in Japanese)

TAKeshita, T., MoRimoto, K., MAO, X., Hashimoto, T. and Furuyama, J. (1993). Phenotypic differences in low $\mathrm{Km}$ aldehyde dehydrogenase in Japanese workers. Lancet 341, 837-838.

Yamada, K., Asaka, A., Norioka, T. and Takeshita, T. (1988). Questionnaire for detecting the phenotype of low $\mathrm{km}$ ALDH (ALDH2). In Biomedical and Social Aspects of Alcohol and Alcoholism, eds Kuriyama, K., Takada, A. and Ishii, H., pp. 481-484. Amsterdam: Excerpta Medica. 